\title{
Productivity growth in OECD countries: A comparison with Malmquist indices
}

\author{
Barış K. Yörük $^{\mathrm{a}, *}$, Osman Zaim ${ }^{\mathrm{b}}$ \\ ${ }^{a}$ Boston College, 140 Commonwealth Avenue, Chestnut Hill, MA 02467-3806, USA \\ b Bilkent University, 06800 Bilkent/Ankara, Turkey \\ Received 4 February 2004; revised 16 February 2005
}

Available online 22 April 2005

Yörük, Barış K., and Zaim, Osman-Productivity growth in OECD countries: A comparison with Malmquist indices

We utilize two alternative indices to measure productivity growth for all but two OECD countries. First, we employ a Malmquist productivity index without considering the existence of hazardous by-products of production processes. To address the shortfalls of this index, we construct an alternative Malmquist-Luenberger productivity index and find that the Malmquist index underestimates the productivity growth. Finally, we investigate the effects of an international protocol on reducing global emissions and country-specific effects on Malmquist-Luenberger productivity measures. Journal of Comparative Economics 33 (2) (2005) 401-420. Boston College, 140 Commonwealth Avenue, Chestnut Hill, MA 02467-3806, USA; Bilkent University, 06800 Bilkent/Ankara, Turkey. (C) 2005 Association for Comparative Economic Studies. Published by Elsevier Inc. All rights reserved.

JEL classification: O40; P52; Q51

Keywords: Malmquist productivity index; Malmquist-Luenberger productivity index; OECD countries

\footnotetext{
* Corresponding author.

E-mail addresses: yoruk@bc.edu (B.K. Yörük), zaim@bilkent.edu.tr (O. Zaim).
}

0147-5967/\$ - see front matter (C) 2005 Association for Comparative Economic Studies. Published by Elsevier Inc. All rights reserved. doi:10.1016/j.jce.2005.03.011 


\section{Introduction}

Increased awareness of environmental quality has prompted policymakers to adopt precise measures of the environmental impacts of policy choices and consider these when formulating economic policy. As environmental issues are becoming more important and treated as international matters, countries are required to measure, document, and publish accurate information on their impact on a set of economic indicators ranging from national accounts to social indicators. As an initial step, an assessment that internalizes negative externalities in production processes is essential. However, traditional measures of productivity growth, e.g., Törnquist and Fischer indices, concentrate only on the production of desirable outputs and fail to consider environmentally hazardous by-products of production processes. Hence, this approach yields biased measures of productivity growth.

To measure productivity growth that accounts for undesirable outputs, one possible approach is to modify traditional indices so as to incorporate negative externalities. However, this methodology requires price information for both desirable and undesirable outputs as well as inputs. In this case, shadow prices for each of various inputs, outputs, and pollutants can be computed by the methods found in Pittman (1983) and Färe et al. (1993). Alternatively, Färe et al. $(1989,1994 a)$ propose a tool to measure productivity that requires information on quantities only. Their non-parametric Malmquist measure relies on constructing a best practice frontier over the whole sample and computing the distance of individual observations from the frontier. This Malmquist index, ${ }^{1}$ hereafter referred to as the $\mathrm{M}$ index, can be partitioned exhaustively into useful component measures. In particular, it can be decomposed into technical change and efficiency change components. However, the $\mathrm{M}$ index must be modified to incorporate negative externalities if environmental issues are to be considered.

In their seminal work, Chung et al. (1997) propose a modified version of the $\mathrm{M}$ index to measure productivity growth in the presence of the joint production of both desirable and undesirable outputs, namely the Malmquist-Luenberger productivity index; hereafter referred to as the ML index. This index considers the reduction of undesirable outputs as well as the increase in desirable outputs; it also possesses all the desirable properties of the $\mathrm{M}$ index. In contrast to the extensive literature on the $\mathrm{M}$ index, only a limited number of empirical studies employ the ML index to measure productivity growth. Using micro-level panel data, Färe et al. (2001) employ the ML index to account for both marketed output and the output of pollution abatement activities of US state manufacturing sectors from 1974 to 1986. Weber and Domazlicky (2001) apply the same methodology to state manufacturing data and the aggregated emissions from the US Environmental Protection Agency's Toxic Release Inventory from 1988 to 1994.

As industrial activity reaches as levels that lead to irreversible environmental damage, governments and international bodies try to enforce regulations to control the resulting pollution. Policies that improve environmental management not only slow the rate of natural resource depletion, but also advance sustainable growth. These standard-setting approaches are referred to as the precautionary principle in Article 3 of United Nations Framework

\footnotetext{
1 The survey chapter in Färe et al. (1998) is an extensive source of references to the literature on the Malmquist productivity indices.
} 
Convention on Climate Change, hereafter referred to as UNFCCC, which aims to reduce global emissions. UNFCCC was negotiated at the Earth Summit in Rio de Janeiro in 1992. The main objective of the convention was to stabilize greenhouse gas concentrations in the atmosphere at desirable levels, but to do so with economic development in a sustainable manner. Along with several mandates, including the Luxembourg Decision of 1990, Rio Summit of 1992, and Berlin Mandate of 1995, UNFCCC has played a key role in establishing a final international agreement, i.e., the Kyoto Protocol of 1997. This protocol is designed to give countries the opportunity to meet the mandated emission targets at low economic cost. Even though the Kyoto Protocol received a worldwide support with 84 signatories, only 64 countries have ratified it as of September 2004. The USA, which accounts for approximately one-third of emissions of highly industrialized Annex 1 countries and onequarter of all global emissions, has refused to ratify the protocol. In addition, two contributors to global emissions, Japan and France, have also refused to ratify the Kyoto Protocol. The lack of participation of these three countries renders the Kyoto Protocol ineffective and makes UNFCCC the primary effective international protocol to date. However, although UNFCCC contains various regulation plans, the mandates are not binding in many aspects.

Using recent macro-level data, our paper contributes to the previous literature by computing and comparing two district indices of productivity growth for each of the OECD countries and by constructing a reliable framework to assess the underlying source of productivity growth. We first compute an $\mathrm{M}$ index to measure the productivity growth of OECD countries and then compute an ML index to incorporate negative externalities. In the absence of information on prices, non-parametric production frontier techniques and distance functions are essential tools for the computation of both indices. These two measures of productivity growth also provide useful information for OECD countries engaged in international protocols, i.e., UNFCCC and the Kyoto Protocol. For example, the precautionary approach of UNFCCC mandates a production plan that is least detrimental to environmental quality. Hence, among the many combinations of inputs, outputs, and pollution emissions, the production plan that maximizes the desirable outputs while simultaneously minimizing undesirable outputs is preferable. To test whether the ML index is a useful measure of compliance with this requirement, we investigate the effects of countryspecific variables and a variable to capture the effect of UNFCCC on the ML index. Our results indicate that the $M$ index underestimates productivity growth and that a threshold level of GDP per capita and industrialization exists for OECD countries, above which an upward trend in productivity growth is observed. Moreover, the UNFCCC variable has a significant and positive effect on the productivity growth measures.

The organization of this paper is as follows. Section 2 presents the trends in emissions for OECD countries, the construction of the indices, and discusses the data sources. Section 3 presents the comparison of the indices. Section 4 discusses the policy implications within a panel data estimation framework. Finally, Section 5 concludes with a summary of the results. We relegate the development of the analytical framework to Appendix A.

\section{Data, trends in emissions, and Malmquist indices}

Along with the percentage change in total emissions from 1983 to 1998, Table 1 reports the percentage change in emission levels of the OECD countries before and after 
Percentage change in emissions and total contribution $(\%)$ of the countries to OECD emissions

\begin{tabular}{|c|c|c|c|c|c|c|c|c|c|c|c|c|c|c|}
\hline & \multicolumn{3}{|c|}{ Change in $\mathrm{CO}_{2}$ emissions } & \multirow{2}{*}{$\begin{array}{l}\text { Total } \\
\text { contri- } \\
\text { bution }\end{array}$} & \multicolumn{3}{|c|}{ Change in $\mathrm{NO}_{x}$ emissions } & \multirow{2}{*}{$\begin{array}{l}\text { Total } \\
\text { contri- } \\
\text { bution }\end{array}$} & \multicolumn{3}{|c|}{ Change in WP emissions } & \multirow{2}{*}{$\begin{array}{l}\text { Total } \\
\text { contri- } \\
\text { bution }\end{array}$} & \multicolumn{2}{|c|}{ Date of ratification } \\
\hline & $1983-91$ & $1992-98$ & $1983-98$ & & 1983-91 & $1992-98$ & $1983-98$ & & 1983-91 & $1992-98$ & $1983-98$ & & UNFCCC & $\begin{array}{l}\text { Kyoto } \\
\text { Protocol }\end{array}$ \\
\hline AUS $^{*}$ & 23.18 & 19.45 & 46.65 & 2.37 & 2.29 & 5.58 & 3.49 & 4.92 & -4.62 & -8.61 & -16.87 & 1.81 & $12 / 30 / 92$ & - \\
\hline AUT $^{*}$ & 14.23 & 6.59 & 16.32 & 0.51 & -9.59 & -10.64 & -23.29 & 0.44 & -9.20 & -14.20 & -24.48 & 0.91 & $02 / 28 / 94$ & $05 / 31 / 02$ \\
\hline $\mathrm{BEL}^{*}$ & 7.21 & 1.07 & 7.40 & 0.91 & -7.48 & -6.44 & -10.92 & 0.77 & -6.49 & -4.96 & -13.41 & 1.18 & $01 / 16 / 96$ & $05 / 31 / 02$ \\
\hline $\mathrm{CAN}^{*}$ & 3.75 & 6.62 & 12.61 & 3.80 & 5.84 & 2.71 & 6.69 & 4.56 & 0.35 & -4.23 & -5.52 & 3.15 & $04 / 21 / 92$ & $12 / 17 / 02$ \\
\hline $\mathrm{DNK}^{*}$ & 20.92 & 5.93 & 8.82 & 0.52 & 22.99 & -65.22 & -63.22 & 0.61 & 41.36 & 12.79 & 55.31 & 0.82 & $12 / 21 / 93$ & $05 / 31 / 02$ \\
\hline FIN $^{*}$ & 21.48 & 21.11 & 34.55 & 0.46 & 11.11 & -10.95 & -3.45 & 0.62 & -14.73 & -14.83 & -32.86 & 0.76 & $05 / 03 / 94$ & $05 / 31 / 02$ \\
\hline FRA $^{*}$ & -5.37 & -6.71 & -16.22 & 3.27 & -0.79 & 4.45 & 1.40 & 3.68 & -7.63 & -9.05 & -17.99 & 6.51 & $03 / 25 / 94$ & - \\
\hline GER ${ }^{*}$ & 2.23 & -2.53 & -3.38 & 7.98 & -22.62 & -22.66 & -45.21 & 5.81 & 1.21 & -7.94 & -2.85 & 8.60 & $12 / 09 / 93$ & $05 / 31 / 02$ \\
\hline GRC $^{*}$ & 19.69 & 14.65 & 50.85 & 0.65 & 15.36 & 11.36 & 28.10 & 0.77 & -3.18 & -3.78 & -8.02 & 0.63 & $08 / 04 / 94$ & $05 / 31 / 02$ \\
\hline HUN $^{*}$ & -21.81 & -18.07 & -43.47 & 0.61 & -23.68 & 6.56 & -26.69 & 0.51 & -12.67 & -18.87 & -35.24 & 1.70 & $02 / 24 / 94$ & - \\
\hline ISL $^{*}$ & 16.11 & 15.89 & 35.94 & 0.02 & 2.56 & 15.00 & 17.95 & 0.05 & -17.09 & -19.08 & -37.88 & 0.07 & $06 / 16 / 93$ & - \\
\hline IRL $^{*}$ & 33.58 & 20.03 & 50.11 & 0.29 & 40.00 & -4.80 & 40.00 & 0.25 & -8.37 & -2.60 & -11.68 & 0.35 & $04 / 20 / 94$ & $05 / 31 / 02$ \\
\hline ITA $^{*}$ & 17.49 & 4.15 & 23.59 & 3.55 & 25.33 & -12.04 & 11.69 & 4.05 & -14.97 & -13.22 & -21.48 & 3.79 & $04 / 15 / 94$ & $05 / 31 / 02$ \\
\hline $\mathrm{JPN}^{*}$ & 26.09 & 10.64 & 41.05 & 9.64 & 1.22 & 0.01 & 1.30 & 3.14 & 7.41 & -4.75 & 1.57 & 15.45 & $05 / 28 / 93$ & - \\
\hline KOR & 88.78 & 45.43 & 200.55 & 2.48 & 18.65 & 32.71 & 91.35 & 2.30 & 18.48 & 2.64 & 19.17 & 3.57 & $12 / 14 / 93$ & $11 / 08 / 02$ \\
\hline LUX $^{*}$ & 30.06 & -6.38 & 23.79 & 0.08 & 58.95 & -6.67 & 47.37 & 0.03 & -16.86 & -15.72 & -31.84 & 0.07 & 05/09/94 & $05 / 31 / 02$ \\
\hline MEX & 9.16 & 7.56 & 25.54 & 2.82 & 0.07 & 0.33 & 0.40 & 3.42 & 32.17 & -16.93 & 7.78 & 1.65 & $03 / 11 / 93$ & $09 / 07 / 00$ \\
\hline NLD $^{*}$ & 25.92 & 9.34 & 36.33 & 1.23 & 2.34 & -18.53 & -18.38 & 1.23 & -0.92 & -9.11 & -11.02 & 1.38 & $12 / 20 / 93$ & - \\
\hline NZL ${ }^{*}$ & 36.47 & 14.29 & 64.03 & 0.22 & 0.71 & 16.45 & 26.43 & 0.34 & -23.84 & -20.58 & -37.61 & 0.50 & $09 / 16 / 93$ & $12 / 19 / 02$ \\
\hline $\mathrm{NOR}^{*}$ & -39.82 & -19.47 & -49.84 & 0.60 & 11.83 & 8.70 & 20.97 & 0.48 & -11.66 & -10.85 & -27.33 & 0.56 & $07 / 09 / 93$ & $05 / 30 / 02$ \\
\hline $\mathrm{POL}^{*}$ & -17.39 & -9.13 & -26.08 & 3.53 & -19.67 & -1.86 & -26.07 & 2.81 & -26.65 & -9.76 & -39.57 & 4.38 & $07 / 28 / 94$ & $12 / 13 / 02$ \\
\hline $\mathrm{PRT}^{*}$ & 41.75 & 16.09 & 82.86 & 0.37 & 282.29 & 7.16 & 336.46 & 0.66 & 45.91 & 8.97 & 51.60 & 1.27 & $12 / 21 / 93$ & - \\
\hline $\mathrm{ESP}^{*}$ & 11.47 & 6.10 & 22.28 & 1.91 & 23.81 & -2.24 & 23.41 & 2.45 & -2.95 & 13.86 & 7.28 & 3.33 & $12 / 21 / 93$ & $05 / 31 / 02$ \\
\hline SWE* & -10.68 & -11.01 & -15.26 & 0.49 & -15.46 & -17.63 & -32.42 & 0.81 & -4.60 & -16.21 & -26.01 & 1.08 & $06 / 23 / 93$ & $05 / 31 / 02$ \\
\hline $\mathrm{CHE}^{*}$ & 5.25 & -0.50 & 6.51 & 0.38 & -9.09 & -21.57 & -31.82 & 0.35 & 5.12 & -6.52 & -7.91 & 1.39 & $12 / 10 / 93$ & $07 / 09 / 03$ \\
\hline TUR* & 47.52 & 28.68 & 93.32 & 1.32 & 45.30 & 44.48 & 112.39 & 1.52 & 7.34 & -1.32 & 12.96 & 1.75 & $02 / 24 / 04$ & - \\
\hline $\mathrm{GBR}^{*}$ & 5.29 & -0.99 & 1.26 & 5.10 & 7.85 & -31.08 & -27.80 & 5.31 & -8.64 & -9.36 & -19.89 & 7.22 & $12 / 08 / 93$ & $05 / 31 / 02$ \\
\hline USA $^{*}$ & 13.98 & 11.61 & 28.73 & 44.89 & 0.16 & 0.19 & 1.50 & 48.10 & -0.45 & 2.09 & 2.58 & 26.11 & $10 / 15 / 92$ & - \\
\hline
\end{tabular}

Notes: (i) The column 'Total contribution' reports a country's total percentage contribution of OECD emissions for the period from 1983 to 1998 . (ii) The country codes are the same as in Table 2.

* An Annex 1 party to the United Nations Framework Convention on Climate Change. 
UNFCCC agreement. By 1994, all but two of the OECD countries had ratified UNFCCC. Belgium ratified the convention in 1996 but Turkey waited until 2004. Interestingly, nine OECD countries have not yet ratified the Kyoto Protocol. Table 1 indicates a considerable variation in emissions across the OECD countries. The USA, Japan, Great Britain, France and Germany are the major contributors of emissions, accounting for more than half of the total emissions from OECD countries. Table 1 reveals the positive impact of UNFCCC on reducing emissions. Almost all OECD countries reduced the growth rate of emissions in the post-UNFCCC period. However, we cannot assess the effect of the Kyoto Protocol on emissions, since the earliest year of ratification is 2000 , which is out of our sample period.

In constructing the M and ML indices, the resource constraint consists of the net fixed standardized capital stock and labor force, measured by the number of employed workers. As the desirable output, we take real GDP measured by purchasing power parity adjusted in 1996 prices. Our proxies for the hazardous by-products ${ }^{2}$ are industrial $\mathrm{CO}_{2}$, i.e., carbon dioxide, $\mathrm{NO}_{x}$, i.e., nitrogen oxide, and organic water pollutant emissions. Data on the capital stock, labor, and real GDP are compiled from a recent data set in Marquetti (2002). World Development Indicators (World Bank, 2002) is the source for $\mathrm{CO}_{2}$ and organic water emissions; whereas data for $\mathrm{NO}_{x}$ emissions are taken from the World Marketing Database (Euromonitor, 2002). The annual panel data set includes 28 OECD countries. The Slovak Republic and the Czech Republic are excluded due to the unavailability of data for these countries. The time period considered is 16 years, from 1983 to 1998.

Initially, we ignore the presence of pollution emissions and compute the $\mathrm{M}$ index by solving the linear programming problem in (6) in Appendix A for each of the OECD countries and for each year considered. In Table 2, we report the cumulative $\mathrm{M}$ index and its decomposition into technical and efficiency changes from 1985 to 1998 by sequential multiplication of the improvements in each year. Values greater than one indicate an improvement in productivity performance, while values less than one imply deterioration. Except for Canada, Japan, Korea, New Zealand, Portugal, Switzerland, and Great Britain, all other OECD countries improved their productivity during the time period. Ireland, Luxembourg, and Finland are the best performers. On average, the OECD countries improved their productivity by almost $3 \%$ between 1985 and 1998. ${ }^{3}$ Table 2 indicates that the main source of productivity growth in the OECD countries is the technical component, which increased by almost $6 \%$ while the efficiency component actually decreased by about $3 \%$. $^{4}$

\footnotetext{
${ }^{2}$ Carbon dioxide and nitrogen oxide emissions from industrial processes result from burning fossil fuels. They include contributions to carbon dioxide and nitrogen oxide produced during the consumption of solid, liquid, gas fuels, and gas flaring. Emissions of organic water pollutants are measured by biochemical oxygen demand, which refers to the amount of oxygen that bacteria in water consume to break down waste. This is a standard water treatment test for the presence of organic pollutants.

3 We computed the M index and decomposed it into the two components for each year and each country. The results are available from the authors upon request.

4 Färe et al. (1994b) compute the M index for 17 OECD countries from 1979 to 1988 and find quite different results. However, our frontier for each year is constructed using the data for 28 OECD countries. Nonetheless, both Färe et al. (1994b) and we determine that the main component of productivity growth in OECD countries is technical change.
} 
Table 2

Cumulative Malmquist productivity Index: 1985 to 1998

\begin{tabular}{|c|c|c|c|c|}
\hline Country code & Malmquist index & Technical change & Efficiency change & Rank \\
\hline AUS & 1.0792 & 1.1296 & 0.9555 & 14 \\
\hline AUT & 1.0767 & 1.1362 & 0.9477 & 15 \\
\hline BEL & 1.0030 & 1.0673 & 0.9398 & 21 \\
\hline CAN & 0.9632 & 1.0822 & 0.8901 & 22 \\
\hline DNK & 1.0741 & 1.1026 & 0.9745 & 16 \\
\hline FIN & 1.4701 & 1.3460 & 1.0925 & 3 \\
\hline FRA & 1.1124 & 1.1442 & 0.9722 & 11 \\
\hline GER & 1.1174 & 1.1466 & 0.9747 & 10 \\
\hline GRC & 1.2583 & 0.9900 & 1.2713 & 6 \\
\hline HUN & 1.0574 & 1.0158 & 1.0412 & 17 \\
\hline ISL & 1.1905 & 1.0990 & 1.0833 & 7 \\
\hline IRL & 1.6419 & 0.9890 & 1.6604 & 1 \\
\hline ITA & 1.1110 & 1.1563 & 0.9610 & 12 \\
\hline JPN & 0.9221 & 1.0061 & 0.9166 & 26 \\
\hline KOR & 0.7514 & 0.9955 & 0.7546 & 28 \\
\hline LUX & 1.4987 & 1.4987 & 1.0000 & 2 \\
\hline MEX & 1.1715 & 1.0128 & 1.1568 & 8 \\
\hline NLD & 1.1209 & 1.1584 & 0.9678 & 9 \\
\hline NZL & 0.9535 & 0.9882 & 0.9651 & 24 \\
\hline NOR & 1.2871 & 1.4898 & 0.8640 & 5 \\
\hline POL & 1.4619 & 1.0416 & 1.4035 & 4 \\
\hline PRT & 0.9366 & 1.0026 & 0.9340 & 25 \\
\hline ESP & 1.0099 & 0.9871 & 1.0231 & 20 \\
\hline SWE & 1.0797 & 0.9855 & 1.0956 & 13 \\
\hline CHE & 0.8850 & 1.4007 & 0.6318 & 27 \\
\hline TUR & 1.0133 & 1.0509 & 0.9645 & 19 \\
\hline GBR & 0.9558 & 0.9921 & 0.9634 & 23 \\
\hline USA & 1.0251 & 1.0303 & 0.9948 & 18 \\
\hline GEOMEAN & 1.0288 & 1.0579 & 0.9727 & N/A \\
\hline
\end{tabular}

Note: The country codes are as follows: AUS: Australia, AUT: Austria, BEL: Belgium, CAN: Canada, DNK: Denmark, FIN: Finland, FRA: France, GER: Germany, GRC: Greece, HUN: Hungary, ISL: Iceland, IRL: Ireland, ITA: Italy, JPN: Japan, KOR: Korea, LUX: Luxembourg, MEX: Mexico, NLD: Netherlands, NZL: New Zealand, NOR: Norway, POL: Poland, PRT: Portugal, ESP: Spain, SWE: Sweden, CHE: Switzerland, TUR: Turkey, GBR: Great Britain, USA: United States.

In constructing the ML indices, we follow Chung et al. (1997) and assume the joint production of good and bad outputs. Although our data set includes information for $\mathrm{CO}_{2}$, $\mathrm{NO}_{x}$, and organic water pollutant emissions, we do not compute an ML index that includes all three. As the number of time periods and variables in the linear programming problem in (9) in Appendix A increases, the number of infeasible solutions is likely to increase. To reduce the number of infeasible solutions in computing the ML index, we follow Färe et al. (2001) and assume that each year's technology is determined by observations on the inputs and outputs of the current and the past two periods. In addition, incorporating negative externalities into adjusted measures of productivity requires assigning weights to bad outputs. Rather than using a contingent valuation, the ML index weighs the relative importance of the bad outputs. Hence, the approach may be interpreted as considering society's preference for the reduction of negative externalities regardless of the actual resulting damage. 
Computing the distance functions in (8) in Appendix A and solving (9) for each period, we calculate four different $\mathrm{ML}$ indices. These indices consider the reduction of only $\mathrm{CO}_{2}$, $\mathrm{NO}_{x}$ plus $\mathrm{CO}_{2}, \mathrm{NO}_{x}$ plus organic water pollutant emissions, and $\mathrm{CO}_{2}$ plus water pollutant emissions, respectively. By multiplying sequentially the improvements in each period, we report the cumulative ML indices and their decompositions into efficiency and technical change for the OECD countries from 1985 to $1998^{5}$ in Table 3. Although the ranking of the countries differ according to the pollutants considered, Ireland and Norway are the best performers across all indices. We find that technical change again dominates the efficiency change in the ML indices. On average, all indices indicate at least $10 \%$ productivity

Table 3

Cumulative Malmquist-Luenberger indices: 1985 to 1998

\begin{tabular}{|c|c|c|c|c|c|c|c|c|c|c|}
\hline \multirow[t]{2}{*}{ Bads } & \multicolumn{5}{|l|}{$\mathrm{CO}_{2}$} & \multicolumn{5}{|c|}{$\mathrm{NO}_{x} / \mathrm{CO}_{2}$} \\
\hline & Index & Efficiency & Technical & Infeas. & Rank & Index & Efficiency & Technical & Infeas. & Rank \\
\hline AUS & 1.0630 & 0.9602 & 1.1070 & - & 19 & 0.9673 & 0.9999 & 0.9674 & 7 & 26 \\
\hline AUT & 1.1265 & 1.0896 & 1.0338 & - & 11 & 1.1063 & 1.0972 & 1.0083 & & 17 \\
\hline BEL & 1.1170 & 1.0001 & 1.1169 & - & 16 & 1.1052 & 0.9618 & 1.1491 & - & 18 \\
\hline CAN & 1.0275 & 0.9284 & 1.1068 & - & 23 & 1.0368 & 0.9542 & 1.0866 & - & 23 \\
\hline DNK & 1.1274 & 1.0165 & 1.1091 & - & 10 & 1.2747 & 1.1532 & 1.1054 & - & 4 \\
\hline FIN & 1.1875 & 1.1591 & 1.0245 & - & 4 & 1.1734 & 1.1737 & 0.9997 & - & 7 \\
\hline FRA & 1.1765 & 1.1386 & 1.0333 & - & 5 & 1.1626 & 1.1162 & 1.0416 & - & 9 \\
\hline GER & 1.1382 & 1.0624 & 1.0713 & - & 8 & 1.1861 & 1.1329 & 1.0469 & - & 5 \\
\hline GRC & 1.1251 & 1.0367 & 1.0852 & - & 12 & 1.1280 & 1.0315 & 1.0935 & - & 13 \\
\hline HUN & 1.1422 & 1.0611 & 1.0765 & - & 7 & 1.1404 & 1.0295 & 1.1078 & - & 11 \\
\hline ISL & 1.1195 & 1.0052 & 1.1137 & - & 15 & 1.0789 & 0.9999 & 1.0790 & 4 & 21 \\
\hline IRL & 1.4668 & 1.3202 & 1.1111 & - & 1 & 1.4395 & 1.2601 & 1.1424 & - & 2 \\
\hline ITA & 1.1006 & 1.0366 & 1.0617 & - & 17 & 1.0944 & 1.0302 & 1.0624 & - & 20 \\
\hline JPN & 0.9820 & 0.9860 & 0.9959 & - & 27 & 1.1762 & 1.0000 & 1.1762 & 5 & 6 \\
\hline KOR & 0.9821 & 0.8716 & 1.1268 & - & 26 & 0.9202 & 0.8824 & 1.0428 & - & 28 \\
\hline LUX & 1.0803 & 1.0000 & 1.0803 & 9 & 18 & 1.0972 & 1.0000 & 1.0972 & 9 & 19 \\
\hline MEX & 1.1232 & 1.0183 & 1.1030 & - & 14 & 1.1189 & 1.0119 & 1.1058 & - & 14 \\
\hline NLD & 1.1292 & 1.0180 & 1.1093 & - & 9 & 1.1551 & 1.0361 & 1.1148 & - & 10 \\
\hline NZL & 0.9404 & 0.8611 & 1.0920 & - & 28 & 0.9422 & 0.8720 & 1.0805 & - & 27 \\
\hline NOR & 1.4088 & 1.2022 & 1.1718 & - & 3 & 1.4607 & 1.2255 & 1.1919 & - & 1 \\
\hline POL & 1.4531 & 1.4062 & 1.0334 & 7 & 2 & 1.3912 & 1.3521 & 1.0289 & 9 & 3 \\
\hline PRT & 0.9879 & 0.8640 & 1.1434 & - & 25 & 1.0005 & 0.8805 & 1.1363 & - & 25 \\
\hline ESP & 1.1246 & 0.9872 & 1.1392 & - & 13 & 1.1101 & 0.9621 & 1.1538 & - & 16 \\
\hline SWE & 1.1672 & 1.0275 & 1.1360 & - & 6 & 1.1697 & 1.0262 & 1.1399 & - & 8 \\
\hline CHE & 1.0134 & 1.0001 & 1.0133 & - & 24 & 1.1316 & 1.0000 & 1.1317 & - & 12 \\
\hline TUR & 1.0578 & 0.9762 & 1.0836 & - & 21 & 1.0710 & 0.9795 & 1.0934 & - & 22 \\
\hline GBR & 1.0558 & 0.9424 & 1.1204 & - & 22 & 1.1127 & 0.9699 & 1.1472 & - & 15 \\
\hline USA & 1.0624 & 0.9985 & 1.0640 & - & 20 & 1.0292 & 1.0000 & 1.0291 & 5 & 24 \\
\hline GEOMEAN & 1.0950 & 1.0284 & 1.0648 & N/A & N/A & 1.1139 & 1.0350 & 1.0762 & N/A & N/A \\
\hline
\end{tabular}

\footnotetext{
${ }^{5}$ For the countries for which some number of infeasible solutions is reported, we use an index for infeasible solutions equal to unity to compute the cumulative productivity growth. Moreover, we computed four ML indices and decomposed them into the two components for each year and each country. The results are available from the authors upon request.
} 
Table 3 (continued)

\begin{tabular}{|c|c|c|c|c|c|c|c|c|c|c|}
\hline \multirow[t]{2}{*}{ Bads } & \multicolumn{5}{|c|}{$\mathrm{NO}_{x} / \mathrm{WP}$} & \multicolumn{5}{|c|}{$\mathrm{CO}_{2} / \mathrm{WP}$} \\
\hline & Index & Efficiency & Technical & Infeas. & Rank & Index & Efficiency & Technical & Infeas. & Rank \\
\hline AUS & 1.0324 & 1.0000 & 1.0324 & 11 & 24 & 1.1927 & 1.0312 & 1.1566 & - & 6 \\
\hline AUT & 1.2853 & 1.0440 & 1.2311 & - & 5 & 1.1735 & 1.0586 & 1.1086 & - & 8 \\
\hline BEL & 1.1073 & 0.8913 & 1.2424 & - & 21 & 1.1170 & 0.9912 & 1.1269 & - & 13 \\
\hline CAN & 1.1587 & 0.9729 & 1.1910 & - & 15 & 1.0811 & 0.9528 & 1.1346 & - & 17 \\
\hline DNK & 1.2689 & 1.1408 & 1.1123 & 1 & 7 & 1.1169 & 1.0250 & 1.0897 & - & 14 \\
\hline FIN & 1.2804 & 1.0612 & 1.2066 & - & 6 & 1.2370 & 1.0925 & 1.1323 & - & 3 \\
\hline FRA & 1.1846 & 0.9535 & 1.2424 & - & 14 & 1.2295 & 1.0874 & 1.1306 & - & 4 \\
\hline GER & 1.2680 & 1.0699 & 1.1852 & - & 8 & 1.1394 & 1.0198 & 1.1173 & - & 11 \\
\hline GRC & 1.1430 & 1.0000 & 1.1431 & - & 17 & 1.1440 & 1.0178 & 1.1240 & - & 10 \\
\hline HUN & 0.8418 & 0.9799 & 0.8591 & 5 & 27 & 0.9066 & 0.9896 & 0.9162 & 6 & 28 \\
\hline ISL & 1.1436 & 1.1736 & 0.9744 & 4 & 16 & 0.9426 & 0.9863 & 0.9557 & 3 & 26 \\
\hline IRL & 1.4308 & 1.2269 & 1.1662 & - & 1 & 1.4472 & 1.2604 & 1.1483 & - & 2 \\
\hline ITA & 1.1953 & 1.0000 & 1.1953 & - & 11 & 1.1738 & 1.0000 & 1.1738 & - & 7 \\
\hline JPN & 1.1241 & 1.0000 & 1.1241 & 4 & 19 & 1.0578 & 0.9457 & 1.1186 & - & 20 \\
\hline KOR & 1.1377 & 0.9732 & 1.1691 & - & 18 & 1.0362 & 0.9499 & 1.0908 & - & 22 \\
\hline LUX & 1.1949 & 1.0000 & 1.1949 & 8 & 12 & 1.0236 & 1.0000 & 1.0236 & 11 & 23 \\
\hline MEX & 1.3858 & 1.0000 & 1.3858 & - & 2 & 1.1619 & 1.0000 & 1.1619 & 6 & 9 \\
\hline NLD & 1.2583 & 1.0163 & 1.2381 & - & 9 & 1.1376 & 1.0209 & 1.1143 & - & 12 \\
\hline NZL & 1.0257 & 0.8980 & 1.1423 & - & 25 & 0.9258 & 0.8606 & 1.0758 & - & 27 \\
\hline NOR & 1.3047 & 1.0228 & 1.2756 & - & 4 & 1.4494 & 1.1957 & 1.2122 & - & 1 \\
\hline POL & 1.3678 & 1.3092 & 1.0447 & 9 & 3 & 1.0175 & 0.9674 & 1.0519 & 8 & 24 \\
\hline PRT & 0.7908 & 0.7763 & 1.0186 & & 28 & 1.0686 & 1.0000 & 1.0686 & & 19 \\
\hline ESP & 1.0728 & 0.8907 & 1.2044 & - & 22 & 1.1068 & 0.9505 & 1.1644 & - & 15 \\
\hline SWE & 1.2179 & 1.0294 & 1.1832 & - & 10 & 1.2071 & 1.0038 & 1.2026 & - & 5 \\
\hline CHE & 1.1120 & 1.0000 & 1.1120 & 7 & 20 & 1.0704 & 1.0000 & 1.0703 & - & 18 \\
\hline TUR & 1.0454 & 0.9742 & 1.0731 & - & 23 & 1.1028 & 0.9859 & 1.1186 & - & 16 \\
\hline GBR & 1.0181 & 0.9805 & 1.0383 & - & 26 & 1.0537 & 0.9355 & 1.1264 & - & 21 \\
\hline USA & 1.1932 & 1.0000 & 1.1932 & - & 13 & 1.0084 & 1.0000 & 1.0084 & 11 & 25 \\
\hline GEOMEAN & 1.2045 & 1.0087 & 1.1941 & N/A & N/A & 1.1062 & 1.0091 & 1.0963 & N/A & N/A \\
\hline
\end{tabular}

Notes: (i) The column labeled 'Infeas.' records the number of infeasible solutions. (ii) The country codes are as follows: AUS: Australia, AUT: Austria, BEL: Belgium, CAN: Canada, DNK: Denmark, FIN: Finland, FRA: France, GER: Germany, GRC: Greece, HUN: Hungary, ISL: Iceland, IRL: Ireland, ITA: Italy, JPN: Japan, KOR: Korea, LUX: Luxembourg, MEX: Mexico, NLD: Netherlands, NZL: New Zealand, NOR: Norway, POL: Poland, PRT: Portugal, ESP: Spain, SWE: Sweden, CHE: Switzerland, TUR: Turkey, GBR: Great Britain, USA: United States.

growth for OECD countries, while the ML index for $\mathrm{NO}_{x}$ and organic water pollutant emissions records a $20 \%$ productivity increase from 1985 to 1998. Finally, in comparison to productivity growth measured by the conventional $\mathrm{M}$ index, these rates are considerably higher.

\section{Comparison of the indices}

If we consider annual sub-periods in which pollutant emissions increase, the measure of productivity growth that explicitly accounts for the joint production of good and bad outputs, i.e., the ML indices, should exhibit slower growth than conventional measures 


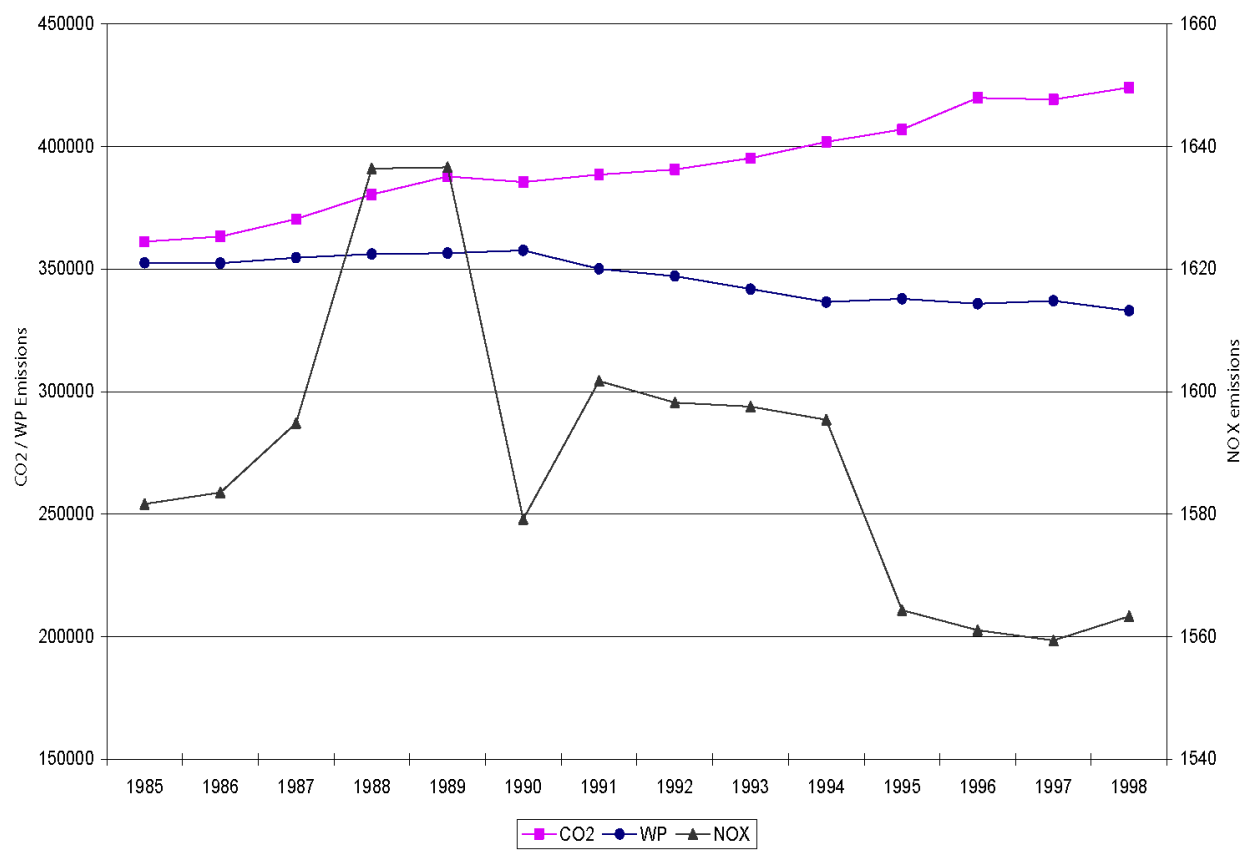

Fig. 1. The trend of pollution emissions in OECD.

that ignore bad outputs, i.e., the $\mathrm{M}$ index. However, this expectation may not hold if the pollution emission pairs that are incorporated in the indices move in opposite directions or a dramatic increase or decrease in the trends of any negative externalities occur during the time period considered. To investigate these issues, we plot pollution emissions for each year as a cumulative average over the OECD countries in Fig. $1 . \mathrm{CO}_{2}$ emissions increase for all years, whereas $\mathrm{NO}_{x}$ and organic water pollutant emissions, denoted by WP, trend upward until 1989 and turn downwards. In Fig. 2, we present the trends in the M and ML indices to investigate their respective movements.

For the subperiod 1985 to 1989, Figs. 1 and 2 support the expectation that as all pollutants exhibit an upwards trend, the $\mathrm{M}$ index tends to overestimate productivity growth compared to the ML indices. For 1990 to 1998, $\mathrm{CO}_{2}$ emissions trend upwards. The M index should again grow faster than the ML index that takes account of $\mathrm{CO}_{2}$. However, Fig. 2 shows that this ML index measures higher productivity growth than does the $M$ index. Moreover, all ML indices exhibit higher productivity growth than the M index during this period for the entire group of countries. Although $\mathrm{CO}_{2}$ emissions increased in almost all OECD countries until 1989, some countries having large weights of $\mathrm{CO}_{2}$ emissions in the sample exhibit a downward trend in these emissions after 1990. In addition, the trends in the ML indices that consider combinations, i.e., $\mathrm{NO}_{x}$ and $\mathrm{CO}_{2}$, and $\mathrm{CO}_{2}$ and $\mathrm{WP}$, may be misleading because $\mathrm{CO}_{2}$ emissions increase while the remaining emissions decrease from 1990 to 1998 . To explore this issue further, we consider two individual countries, each in turn. 


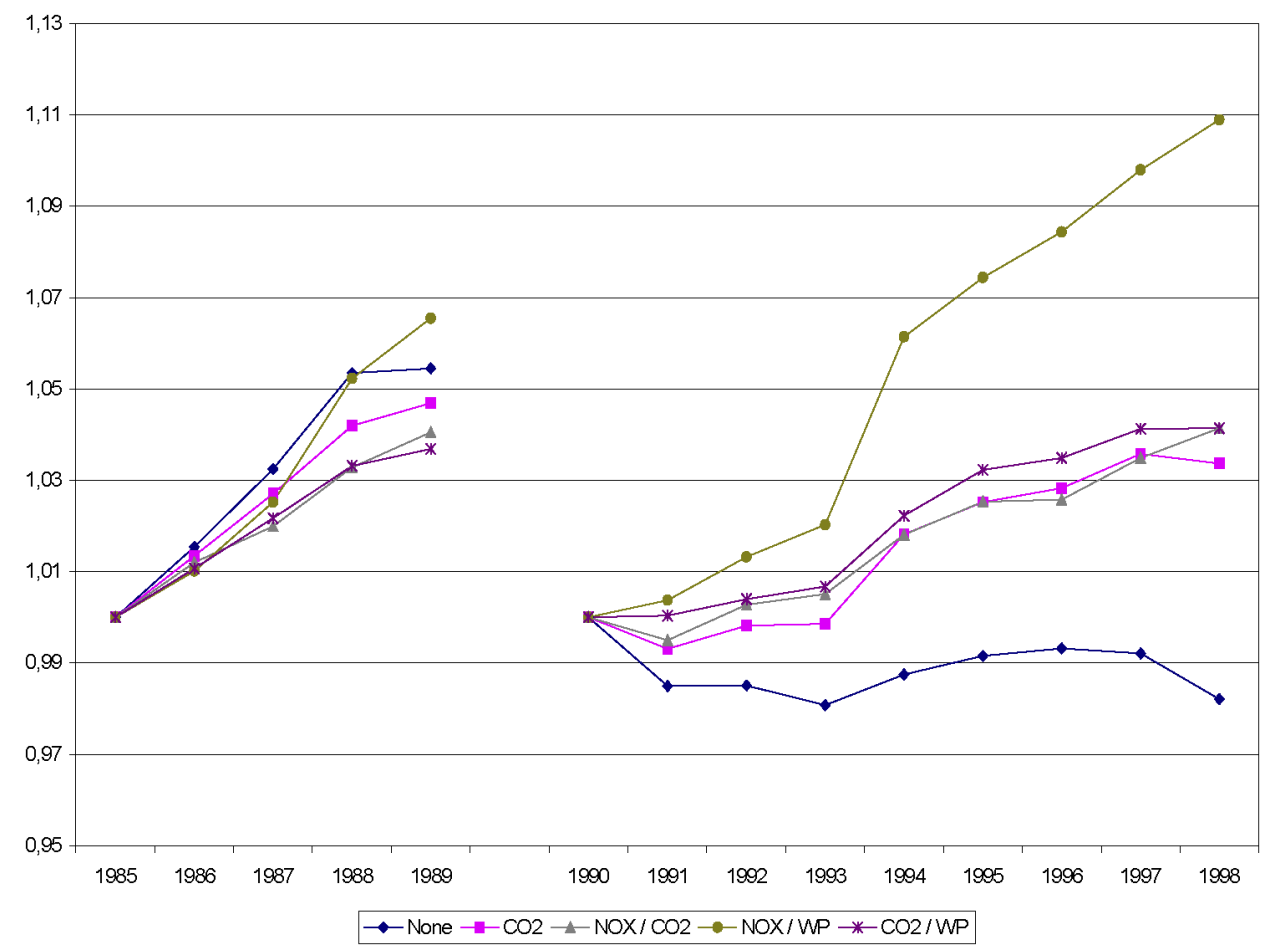

Fig. 2. The trend of indices for OECD.

Figure 3 records the annual trends of pollution emissions for Great Britain. $\mathrm{NO}_{x}$ emissions increase until 1989, while organic water pollutant and $\mathrm{CO}_{2}$ emissions stay almost constant over this period. For the remaining years, $\mathrm{NO}_{x}$ and $\mathrm{WP}$ emissions trend downward, while $\mathrm{CO}_{2}$ emissions continue to exhibit a steady path. Figure 4 plots the trends of the M and ML indices for Great Britain. Up to 1989, the M index measures higher productivity growth than any of the ML indices, due to the significant upward trend of $\mathrm{NO}_{x}$ emissions during period. As a response to the small decline in $\mathrm{CO}_{2}$ emissions between 1987 and 1988, the ML index that takes account of $\mathrm{CO}_{2}$ emissions dominates the $\mathrm{M}$ index. For the remaining years, from 1989 to 1998, the ML indices exhibit higher productivity growth than does the $\mathrm{M}$ index. This result is expected because the pollutant emissions for Great Britain trend downward during this period.

Norway is one the best performers in all indices. Figure 5 plots the trends of pollutants in Norway from 1985 to 1998. Organic water pollutant emissions have a downward trend for most of this period, while $\mathrm{NO}_{x}$ emissions decrease until 1992 and trend upwards for the remaining years. $\mathrm{CO}_{2}$ emissions fluctuate over time in Norway, declining until 1989, and then increasing between 1989 and 1996. The trends of the M and ML indices for Norway are presented in Fig. 6 divided into four different subperiods. ${ }^{6}$ In response to

\footnotetext{
${ }^{6}$ For clarity, the indices are normalized to unity with respect to fluctuations in pollution emission trends.
} 


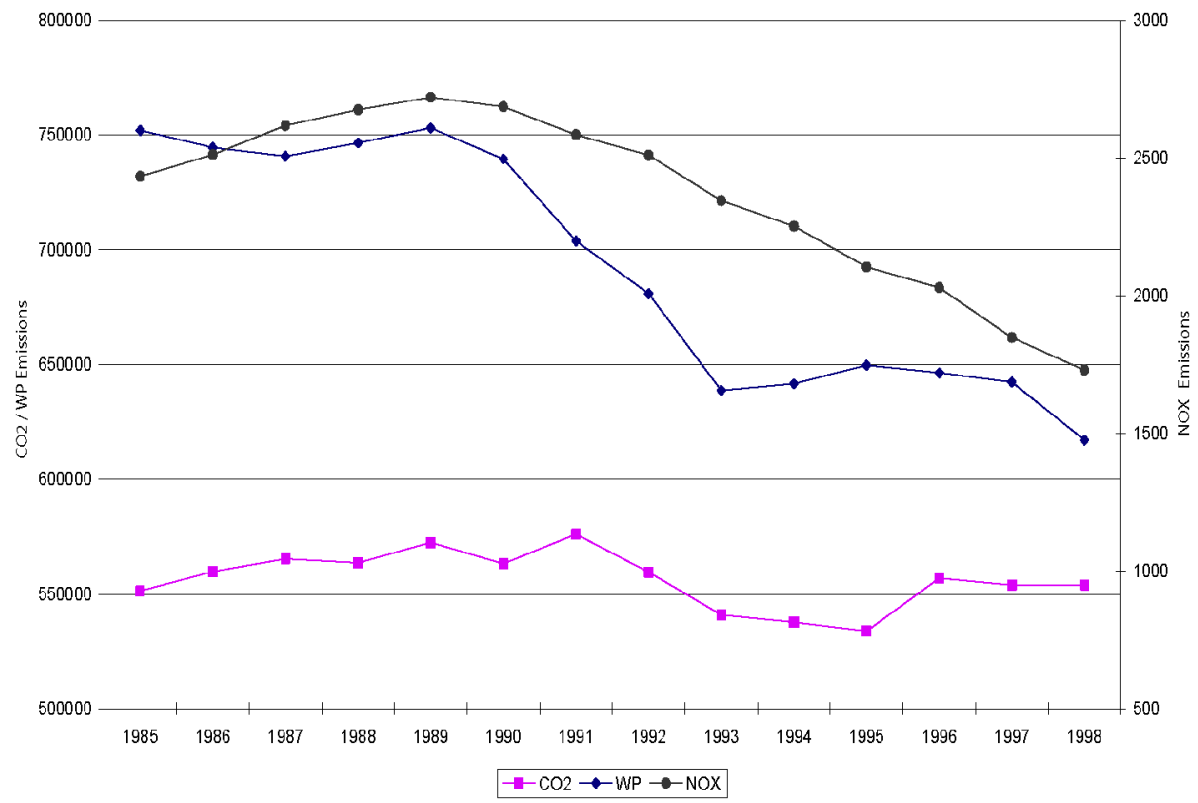

Fig. 3. The trend of pollution emissions in Great Britain.

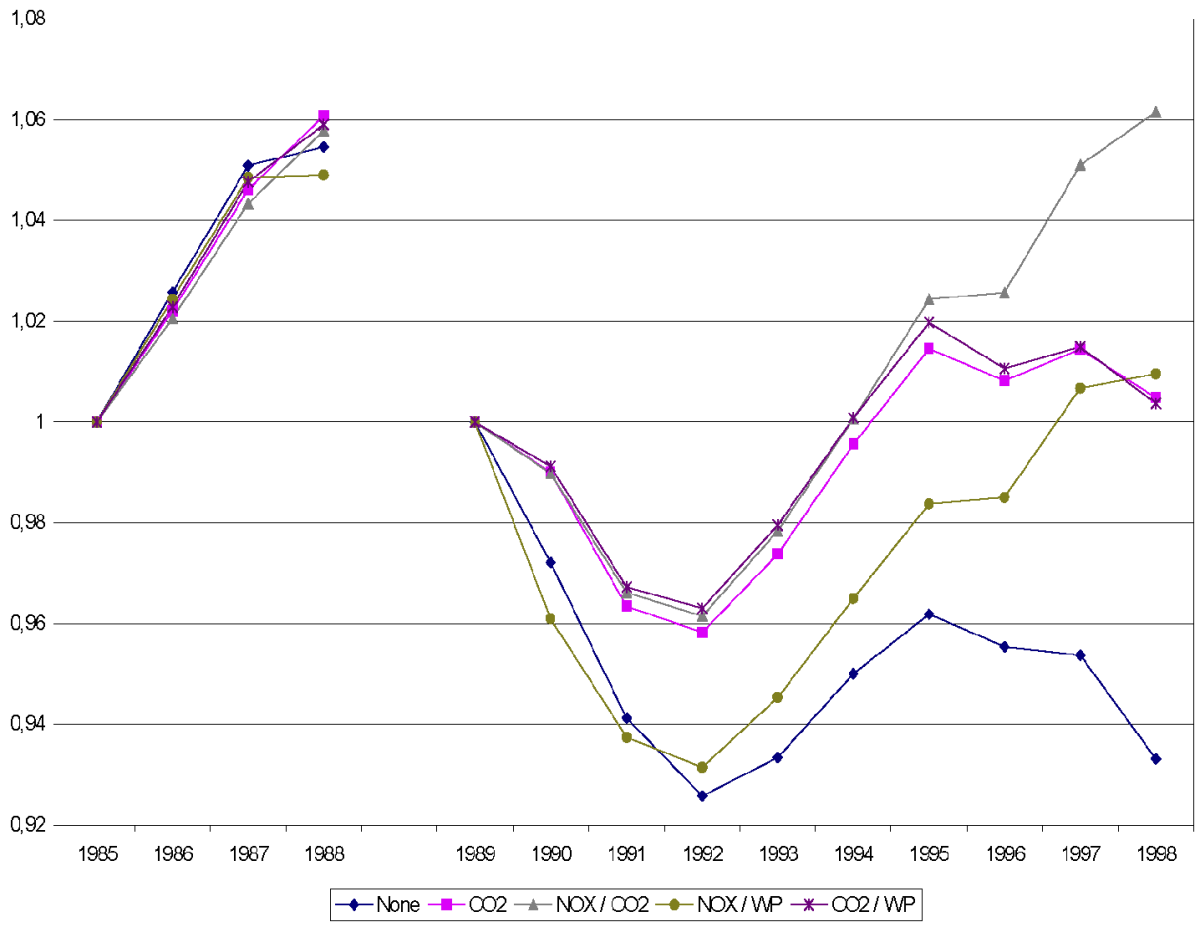

Fig. 4. The trend of indices for Great Britain. 


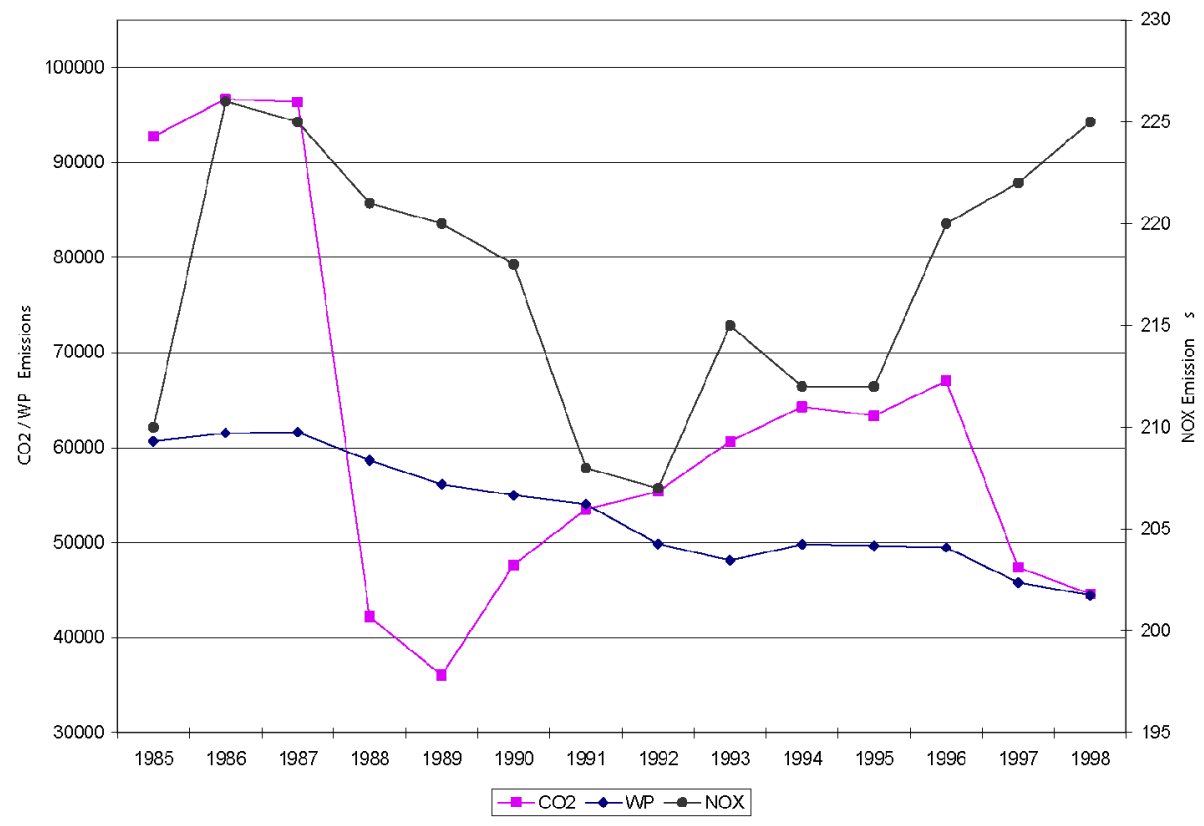

Fig. 5. The trend of pollution emissions in Norway.

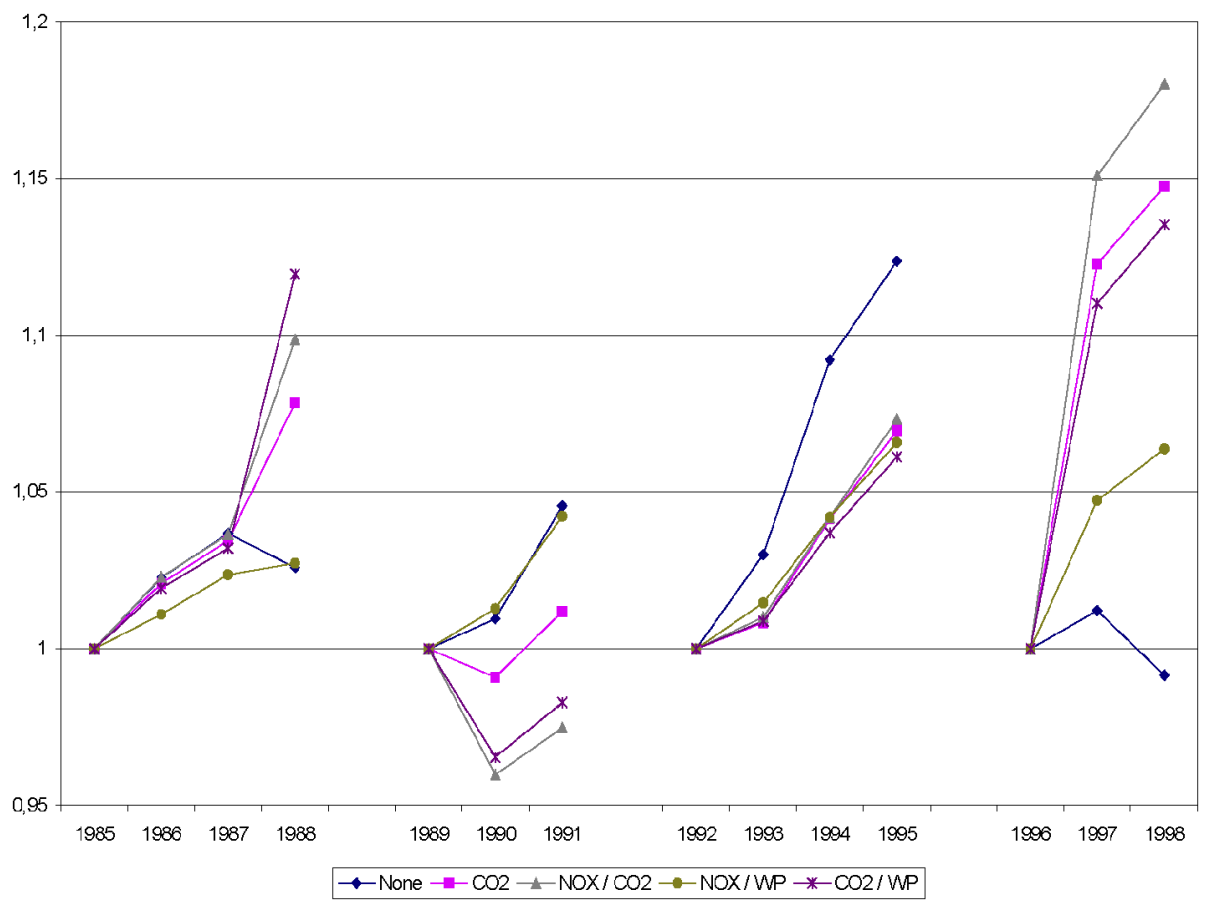

Fig. 6. The trend of indices for Norway. 
an upward trend in all emissions, the $\mathrm{M}$ index dominates the ML indices in Norway for the subperiod from 1985 to 1987. Due to a dramatic decline in $\mathrm{CO}_{2}$ emissions, the $\mathrm{ML}$ index that accounts for these pollutants measure higher productivity growth than the $\mathrm{M}$ index between 1987 and 1988. In the next subperiod from 1989 to $1991, \mathrm{NO}_{x}$ and organic water pollutant emissions decrease. As expected, the ML index that accounts for both of these pollutants measures higher productivity growth than the $\mathrm{M}$ index during this period. From 1992 to $1995, \mathrm{NO}_{x}$ and $\mathrm{CO}_{2}$ emissions increase dramatically. As a response, the $\mathrm{M}$ index measures higher productivity growth than all the ML indices. Finally, the decline in organic water pollutant and $\mathrm{CO}_{2}$ emissions from 1995 to 1998 is captured by the ML indices dominating the $\mathrm{M}$ index during this time.

From the detailed analysis of two individual countries, we find that, during periods for which countries' emissions trend upwards, the M index measures higher productivity growth than the ML indices. Hence, the M index overestimates productivity growth in these situations. During time periods in which emissions trend downwards, the ML indices exhibit higher productivity growth than the $\mathrm{M}$ index. Hence, the $\mathrm{M}$ index underestimates productivity growth in this situation. Therefore, we conclude that the $\mathrm{M}$ index is a biased measure of productivity growth and that information on emissions should be used to construct a more accurate measure of productivity growth. ${ }^{7}$

\section{Empirical results}

Having established that the ML indices are the preferred productivity measure, we investigate effects of country-specific variables and a variable capturing the UNFCCC protocol on productivity growth for these OECD countries. In our panel regression framework, the dependent variable is the ML index and the explanatory variables are real GDP per capita (GDPPC), the share of industry in GDP (INDS), and UNFCCC, which is a dummy variable that takes the value of one for the year in which the sample country ratified the UNFCCC and all subsequent years. ${ }^{8}$ The squares of both GDP per capita and the share of industry in GDP are included to capture any quadratic relationships between the ML index and these variables. Data for GDP per capita and the share of industry in GDP are taken from the World Development Indicators (World Bank, 2002). We take the ML index that accounts for $\mathrm{CO}_{2}$ emissions as the dependent variable in Table 4 and provide the parameter estimates of the explanatory variables under fixed-effects and random-effects specifications, both with and without the industry share in GDP.

The Hausman test indicates that the fixed-effects specification is preferred for both sets of regressions. All of the parameter estimates are statistically significant. The quadratic relationship between the ML index that accounts for $\mathrm{CO}_{2}$ emissions and real GDP is U-type with a turning point at approximately $\$ 24,300$. Hence, once an OECD country reaches

\footnotetext{
7 Both a simple $t$-test and non-parametric tests allow us to reject the null hypothesis that the means of the M and $\mathrm{ML}$ indices are the same for only the ML index that accounts for $\mathrm{CO}_{2}$ emissions at conventional significance levels. We were unable to reject the null hypothesis for the other three indices.

${ }^{8}$ Färe et al. (2001) present a similar regression with a regulation dummy in their analysis of state manufacturing growth.
} 
Table 4

Parameter estimates for the ML index $\left(\mathrm{CO}_{2}\right)$

\begin{tabular}{|c|c|c|c|c|}
\hline & \multicolumn{2}{|c|}{ Without industry share } & \multicolumn{2}{|c|}{ With industry share } \\
\hline & Fixed effects & Random effects & Fixed effects & Random effects \\
\hline Constant & $\begin{array}{l}1.097^{* *} \\
(0.016)\end{array}$ & $\begin{array}{l}1.037^{* *} \\
(0.095)\end{array}$ & $\begin{array}{c}1.238^{* *} \\
(0.0719)\end{array}$ & $\begin{array}{l}1.069^{* *} \\
(0.058)\end{array}$ \\
\hline GDPPC & $\begin{array}{c}-9.37 \mathrm{E}-06^{* *} \\
(1.88 \mathrm{E}-06)\end{array}$ & $\begin{array}{c}-3.29 \mathrm{E}-06^{* *} \\
(1.19 \mathrm{E}-06)\end{array}$ & $\begin{array}{c}-9.87 \mathrm{E}-06^{* * *} \\
(1.85 \mathrm{E}-06)\end{array}$ & $\begin{array}{c}-3.82 \mathrm{E}-06^{* *} \\
(1.28 \mathrm{E}-06)\end{array}$ \\
\hline GDPPC $^{2}$ & $\begin{array}{l}1.95 \mathrm{E}-10^{* *} \\
(4.97 \mathrm{E}-11)\end{array}$ & $\begin{array}{c}7.93 \mathrm{E}-11^{*} \\
(3.58 \mathrm{E}-11)\end{array}$ & $\begin{array}{l}2.00 \mathrm{E}-10^{* *} \\
(4.90 \mathrm{E}-11)\end{array}$ & $\begin{array}{c}9.95 \mathrm{E}-11^{* * *} \\
(3.83 \mathrm{E}-11)\end{array}$ \\
\hline INDS & - & - & $\begin{array}{c}-0.803^{*} \\
(0.381)\end{array}$ & $\begin{array}{r}-0.229 \\
(0.336)\end{array}$ \\
\hline INDS $^{2}$ & - & - & $\begin{array}{c}1.172^{*} \\
(0.531)\end{array}$ & $\begin{array}{c}0.418 \\
(0.478)\end{array}$ \\
\hline Protocol & $\begin{array}{l}0.0124^{* *} \\
(0.0035)\end{array}$ & $\begin{array}{c}0.0041 \\
(0.0029)\end{array}$ & $\begin{array}{l}0.0131^{* *} \\
(0.0036)\end{array}$ & $\begin{array}{c}0.0054 \\
(0.0031)\end{array}$ \\
\hline Turning point (GDPPC) & 24,026 & 20,744 & 24,675 & 19,196 \\
\hline Turning point (INDS) & - & - & 0.34 & 0.27 \\
\hline Hausman test & - & 21.51 & - & 29.64 \\
\hline$R^{2}$ & 0.092 & 0.072 & 0.124 & 0.079 \\
\hline Number of observations & 346 & 346 & 333 & 333 \\
\hline
\end{tabular}

Notes: (i) The values in parenthesis are standard errors. (ii) The Hausman test indicates that the fixed-effects specification is preferred in both cases.

* Significance at the $5 \%$ level.

** Significance at the $1 \%$ level.

this threshold income level, an upward trend in productivity growth is observed. Table 4 indicates the same quadratic relationship between the ML index and the share of industry in GDP, with the threshold level of industrialization at $34 \%$. Hence, once the share of total industry in GDP exceeds $34 \%$ for an OECD country, productivity growth trends upwards. Finally, the coefficient of the dummy variable is positive and highly significant in both preferred specifications. Therefore, we find strong empirical support for a positive impact of UNFCCC on productivity growth in OECD countries that have ratified the convention.

Although we do not report the results, we ran the same regressions with the other three ML indices as dependent variables. ${ }^{9}$ The results are virtually equivalent except for the index accounting for both $\mathrm{NO}_{x}$ and water pollutant emissions. For that regression, the random effects model is the preferred specification and all coefficients are statistically insignificant. Finally, we ran the regression using the $M$ index as the dependent variable and found all the coefficients to be statistically insignificant. Hence, we conclude that international regulations have a considerable impact on productivity growth measures that account for negative externalities but have no significant effect on conventional Malmquist measures.

\footnotetext{
9 The results are available from the authors upon request.
} 


\section{Conclusion}

The OECD has a long-standing program to improve resource efficiency, to address the environmental impact of growth, and to consider issues related to technological change. Efficient use of resources encourages growth and sustainable development in OECD countries. However, measures that internalize negative externalities in production processes are required to provide an accurate assessment of environmental problems. Following Färe et al. (1994b) and Chung et al. (1997), we measure productivity growth of the OECD countries using two indices, namely the Malmquist (M) index and the Malmquist-Luenberger (ML) index. We find that the $\mathrm{M}$ index, which does not account for negative externalities, measures higher productivity growth than the ML index during the periods in which undesirable outputs trend upwards. Alternatively, during time periods exhibiting a downward trend in pollutants, the ML index is larger than the M index. Therefore, we conclude that the $\mathrm{M}$ index is not well-suited to measure productivity in the presence of negative externalities.

Although the ranking of countries differs according to which emissions are included, Ireland and Norway are the best performers for all four ML indices computed. In addition, the technical change component dominates the efficiency change component in these ML indices. The ML indices measure average productivity growth of at least about $10 \%$ for the OECD countries from 1985 to 1998 , with the index that includes nitrogen oxide and organic water pollutant emissions, indicating a $20 \%$ productivity growth. Compared with the conventional $\mathrm{M}$ index, the ML indices record at least $7 \%$ higher productivity growth for OECD countries. Finally, we investigate the determinants of the variation in productivity growth across these countries, paying attention to the potential role played by the UNFCCC protocol on emissions. We find that the dummy variable representing the ratification of this agreement has a significant, positive effect on the ML index. Furthermore, we establish threshold levels of GDP per capita and industrialization for the OECD countries above which productivity growth trends upward.

\section{Acknowledgments}

Frank Gollop, Syed F. Mahmud, Süheyla Özyıldırım, Asel Aliyosova, three anonymous referees, and the Editor provided helpful comments. The authors take sole responsibility for the contents of the paper.

\section{Appendix A. The analytical framework}

To describe the theoretical foundation of our model, we denote desirable or good outputs as a vector $y=\left(y_{1}, \ldots, y_{M}\right) \in R_{+}^{M}$ and undesirable or bad outputs as a vector $b=\left(b_{1}, \ldots, b_{I}\right) \in R_{+}^{I}$. The output set $(y, b)$ is produced by the input vector $x=$ $\left(x_{1}, \ldots, x_{N}\right) \in R_{+}^{N}$ and technology is described as

$$
T=\{(x, y, b): x \text { can produce }(y, b)\} .
$$


For each input vector $x=\left(x_{1}, \ldots, x_{N}\right) \in R_{+}^{N}$, the technology set includes all the combinations of good and bad outputs, i.e., the output set $(y, b)$ that can be produced by the vector of inputs. The technology set is equivalent to an output set $P(x)$ and input set $L(y, b)$ such that

$$
(x, y, b) \in T \quad \Leftrightarrow \quad(y, b) \in P(x) \quad \Leftrightarrow \quad x \in L(y, b) .
$$

A weak disposability assumption, which implies that, for a given fixed level of inputs, a proportional reduction in goods and bads is always feasible ${ }^{10}$ is specified as

$$
(y, b) \in P(x) \text { and } 0 \leqslant \theta \leqslant 1 \quad \text { imply } \quad(\theta y, \theta b) \in P(x) .
$$

In addition, the assumption of free disposability of good outputs asserts that good outputs can be reduced without a corresponding reduction of bad outputs. Hence, we have:

$$
(y, b) \in P(x) \text { and } y^{\prime} \leqslant y \quad \text { imply } \quad\left(y^{\prime}, b\right) \in P(x) .
$$

Equations (1) and (2) model the asymmetry between good and bad outputs in that goods are freely disposable but bads are not. The final assumption of null-jointness implies that no desirable outputs can be produced without producing any undesirable outputs. The joint production of good and bad outputs is specified as

$$
\text { if } \quad(y, b) \in P(x) \text { and } b=0, \quad \text { then } \quad y=0 .
$$

In addition to these assumptions, we impose some restrictions on the output set $P(x)$. First, no output is producible without inputs, so that we have:

$$
P(0)=\{0,0\} .
$$

Second, given a finite number of inputs, only a finite number of outputs can be produced so that we require $P(x)$ to be a compact set for each $x \in R_{+}^{N}$. Finally, we impose free disposability of inputs so that, if inputs are increased, outputs do not decrease. Hence, we have

$$
P(x) \supseteq P\left(x^{\prime}\right), \quad x \geqslant x^{\prime} .
$$

Following Färe et al. (1994b), we use data envelopment analysis, hereafter, DEA. We assume a total of $K$ observations on inputs and outputs and let $k$ index each individual observation so that we specify $\left\{\left(x^{k}, y^{k}, b^{k}\right): k=1, \ldots, K\right\}$. With this information, we construct an output set that holds for every period and satisfies our assumptions. Formally, we have:

$$
\begin{aligned}
P(x)=\{(y, b): & \sum_{k=1}^{K} z_{k} y_{k m} \geqslant y_{m}, m=1, \ldots, M, \sum_{k=1}^{K} z_{k} b_{k i}=b_{i}, i=1, \ldots, I, \\
& \left.\sum_{k=1}^{K} z_{k} x_{k n} \leqslant x_{n}, n=1, \ldots, N, \text { and } z_{k} \geqslant 0, k=1, \ldots, K\right\},
\end{aligned}
$$

\footnotetext{
10 We thank an anonymous referee for the interpretation. Shephard and Färe (1974) provide detailed explanations of the assumptions for production frontiers.
} 
where $z_{k}$ are non-negative intensity variables or weights assigned to each observation to construct the production set. The inequality constraint on good outputs $y=\left(y_{1}, \ldots, y_{M}\right) \in$ $R_{+}^{M}$ in (3) represents the assumption of free disposability, which means that the desirable output can be disposed of without the use of any inputs.

Because the production of undesirable outputs $b=\left(b_{1}, \ldots, b_{I}\right) \in R_{+}^{I}$ accompanies the production of desirable outputs, we must impose a weak disposability condition similar to (1) by choosing an equality sign for the relevant constraint. To satisfy the assumption that the good and bad outputs are null-joint, we impose the following conditions:

$$
\begin{aligned}
& \sum_{k=1}^{K} b_{k i}>0, \quad i=1, \ldots, I, \quad \text { and } \\
& \sum_{i=1}^{I} b_{k i}>0, \quad i=1, \ldots, K .
\end{aligned}
$$

Inequality (4) states that each undesirable output is produced by some individual observation $k$, i.e., firm or country. Moreover, Eq. (5) implies that every $k$ produces at least one unit of bad output.

To illustrate null-jointness further, we assume that each $b_{i}=0$, where $i=1, \ldots, I$. Then each intensity variable $z_{k}$ in (3) is zero, which means that all desirable outputs must be zero. Therefore, these two restrictions determine whether a particular data set satisfies the null-jointness assumption for both desirable and undesirable outputs. In our application, we exclude the data that violate the null-jointness assumption. Furthermore, the non-negativity of intensity variables in (3) implies that the production technology exhibits constants returns to scale. Hence, we have:

$$
P(\lambda x)=\lambda P(x), \quad \lambda>0 .
$$

Since we have no information on prices, we use distance functions as proxies for defining and measuring productivity growth.

The original $\mathrm{M}$ index uses distance functions to represent the underlying technology following Shephard (1970). If all outputs are desirable, these output distance functions are defined as

$$
D_{o}(x, y)=\inf \left\{\theta:\left(x, \frac{y}{\theta}\right) \in P(x)\right\},
$$

which provides complete characterization of the technology. For each observation, output distance functions can be computed by solving the following linear programming problem for $k^{\prime}$ :

$$
\begin{array}{ll}
\left(D_{o}^{t}\left(x^{t, k^{\prime}}, y^{t, k^{\prime}}\right)\right)^{-1}=\max \theta \\
\text { s.t. } \quad \sum_{k=1}^{K} z_{k} y_{k m}^{t} \geqslant \theta y_{k^{\prime} m}^{t}, \quad m=1, \ldots, M, \\
& \sum_{k=1}^{K} z_{k} x_{k n}^{t} \leqslant x_{k^{\prime} n}^{t}, \quad n=1, \ldots, N,
\end{array}
$$




$$
z_{k} \geqslant 0, \quad k=1, \ldots, K
$$

Denoting $t=1, \ldots, T$ as the time periods, we define an output-oriented $\mathrm{M}$ index without the bad outputs following Färe et al. (1989). Hence, we have:

$$
M_{o}\left(x^{t}, y^{t}, x^{t+1}, y^{t+1}\right)=\left[\frac{D_{o}^{t}\left(x^{t+1}, y^{t+1}\right)}{D_{o}^{t}\left(x^{t}, y^{t}\right)} \frac{D_{o}^{t+1}\left(x^{t+1}, y^{t+1}\right)}{D_{o}^{t+1}\left(x^{t}, y^{t}\right)}\right]^{1 / 2} .
$$

The $\mathrm{M}$ index can be decomposed into two components, an efficiency change, i.e., $M E F F C H$, and a technical change, i.e., MTECH. These components are defined as

$$
\begin{aligned}
& \text { MEFFCH }=\frac{D_{o}^{t+1}\left(x^{t+1}, y^{t+1}\right)}{D_{o}^{t}\left(x^{t}, y^{t}\right)} \quad \text { and } \\
& M T E C H=\left[\frac{D_{o}^{t}\left(x^{t+1}, y^{t+1}\right)}{D_{o}^{t+1}\left(x^{t+1}, y^{t+1}\right)} \frac{D_{o}^{t}\left(x^{t}, y^{t}\right)}{D_{o}^{t+1}\left(x^{t}, y^{t}\right)}\right]^{1 / 2} .
\end{aligned}
$$

The Malmquist productivity measure is simply the product of these two components. That is:

$$
M_{t}^{t+1}=\mathrm{MEFFCH}_{t}^{t+1} \cdot \mathrm{MTECH}_{t}^{t+1} .
$$

This $\mathrm{M}$ index has several desirable features. Unlike other measures such as the Fischer and the Törnquist indices, the $\mathrm{M}$ index does not require price information for outputs and inputs. Although the $\mathrm{M}$ index can be used to measure productivity in the presence of bad outputs, the underlying distance functions do not adjust individual observations for negative externalities. To account for bad outputs in a productivity measure, the output distance functions can be characterized by

$$
D_{o}(x, y, b)=\inf \{\theta:((y, b) / \theta) \in P(x)\} .
$$

However, without taking account of the reduction attributable to bad outputs, the output distance function in (7) expands the desirable and undesirable output set $(y, b)$ proportionally as much as it is feasible. ${ }^{11}$

The ML index is a modified version of the M index. Rather than using Shephard's output distance functions, the ML index takes directional output distance functions to represent the underlying technology. This approach takes account of the reduction attributable to undesirable outputs and includes credits for the increase in desirable outputs. Following Chung et al. (1997), we formulate directional distance functions as

$$
\bar{D}_{o}(x, y, b ; g)=\sup \{\beta:(y, b)+\beta g \in P(x)\},
$$

where $g$ is the vector of directions, ${ }^{12}$ which may be defined as $g=(y,-b)$. In a similar manner to Shephard's distance functions, directional distance functions can be computed as a solution to a set of linear programming problems. We formalize such a problem for $k^{\prime}$

\footnotetext{
11 Chung et al. (1997) provide further discussion of this issue.

12 This given vector of directions is only one possibility. Defined in this manner, we can easily compare the ML index with the $\mathrm{M}$ index without bad outputs. When the direction $g$ is $(y, b)$ rather than $(y,-b)$, the two indices coincide.
} 
as

$$
\begin{array}{ll} 
& \bar{D}_{o}^{t}\left(x^{t, k^{\prime}}, y^{t, k^{\prime}}, b^{t, k^{\prime}} ; y^{t, k^{\prime}},-b^{t, k^{\prime}}\right)=\max \beta \\
\text { s.t. } & \sum_{k=1}^{K} z_{k} y_{k^{\prime} m}^{t} \geqslant(1+\beta) y_{k^{\prime} m}^{t}, \quad m=1, \ldots, M, \\
& \sum_{k=1}^{K} z_{k} b_{k i}^{t}=(1-\beta) b_{k^{\prime} i}^{t}, \quad i=1, \ldots, I, \\
& \sum_{k=1}^{K} z_{k} x_{k n}^{t} \leqslant(1-\beta) x_{k^{\prime} n}^{t}, \quad n=1, \ldots, N, \\
& z_{k} \geqslant 0, \quad k=1, \ldots, K .
\end{array}
$$

Letting $g=(y,-b)$, the output-oriented ML index is given by

$$
\begin{aligned}
M L_{t}^{t+1}= & {\left[\frac{1+\bar{D}_{o}^{t}\left(x^{t}, y^{t}, b^{t} ; y^{t},-b^{t}\right)}{1+\bar{D}_{o}^{t}\left(x^{t+1}, y^{t+1}, b^{t+1} ; y^{t+1},-b^{t+1}\right)}\right.} \\
& \left.\times \frac{1+\bar{D}_{o}^{t+1}\left(x^{t}, y^{t}, b^{t} ; y^{t},-b^{t}\right)}{1+\bar{D}_{o}^{t+1}\left(x^{t+1}, y^{t+1}, b^{t+1} ; y^{t+1},-b^{t+1}\right)}\right]^{1 / 2} .
\end{aligned}
$$

This index can also be decomposed into two components. The efficiency component is given as

$$
\operatorname{MLEFFCH}_{t}^{t+1}=\frac{1+\bar{D}_{o}^{t}\left(x^{t}, y^{t}, b^{t} ; y^{t},-b^{t}\right)}{1+\bar{D}_{o}^{t+1}\left(x^{t+1}, y^{t+1}, b^{t+1} ; y^{t+1},-b^{t+1}\right)}
$$

and the technical change component can be written as

$$
\begin{aligned}
\operatorname{MLTECH}_{t}^{t+1}= & {\left[\frac{1+\bar{D}_{o}^{t+1}\left(x^{t}, y^{t}, b^{t} ; y^{t},-b^{t}\right)}{1+\bar{D}_{o}^{t}\left(x^{t}, y^{t}, b^{t} ; y^{t},-b^{t}\right)}\right.} \\
& \left.\times \frac{1+\bar{D}_{o}^{t+1}\left(x^{t+1}, y^{t+1}, b^{t+1} ; y^{t+1},-b^{t+1}\right)}{1+\bar{D}_{o}^{t}\left(x^{t+1}, y^{t+1}, b^{t+1} ; y^{t+1},-b^{t+1}\right)}\right]^{1 / 2} .
\end{aligned}
$$

Finally, the ML index is equal to the product of these two components, that is:

$$
M L_{t}^{t+1}=M L E F F C H_{t}^{t+1} \cdot M L T E C H_{t}^{t+1} .
$$

\section{References}

Chung, Yi H., Färe, Rolf, Grosskopf, Shawna, 1997. Productivity and undesirable outputs: A directional distance function approach. Journal of Environmental Management 51, 229-240.

Euromonitor, 2002. World Marketing Database. Data set.

Färe, Rolf, Grosskopf, Shawna, Valdmanis, Vivian, 1989. Capacity, competition and efficiency in hospitals: A non-parametric approach. Journal of Productivity Analysis 1, 123-128. 
Färe, Rolf, Grosskopf, Shawna, Lovell, Knox C.A., Yaisawarng, Suthathip, 1993. Derivation of shadow prices for undesirable outputs: A distance function approach. Review of Economics and Statistics 75, 374-380.

Färe, Rolf, Grosskopf, Shawna, Lovell, Knox C.A., 1994a. Production Frontiers. Cambridge Univ. Press, Cambridge, UK.

Färe, Rolf, Grosskopf, Shawna, Norris, Mary, Zhang, Zhongyang, 1994b. Productivity growth technical progress and efficiency change in industrialized countries. American Economic Review 84, 66-83.

Färe, Rolf, Grosskopf, Shawna, Roos, Pontus, 1998. Malmquist productivity indexes: A survey of theory and practice. In: Russell, Robert R., Färe, Rolf, Grosskopf, Shawna (Eds.), Index Number Theory Essays in Honor of Sten Malmquist. Kluwer Academic, Boston, pp. 127-190.

Färe, Rolf, Grosskopf, Shawna, Pasurka, Carl, 2001. Accounting for air pollution emissions in measuring state manufacturing productivity growth. Journal of Regional Science 41, 381-409.

Marquetti, Adalmir, 2002. Extended Penn World Tables 2.0 Data set. Available at http://homepage.newschool. edu/ foleyd/epwt.

Pittman, Russell W., 1983. Multilateral productivity comparisons with undesirable outputs. Economic Journal 93, 883-891.

Shephard, Ronald W., 1970. Theory of Cost and Production Frontiers. Princeton Univ. Press, Princeton NJ.

Shephard, Ronald W., Färe, Rolf, 1974. Laws of diminishing returns. Zeitschrift für Nationalökonomie 34, 69-90.

Weber, William, Domazlicky, Bruce, 2001. Productivity growth and pollution in state manufacturing. Review of Economics and Statistics 83, 195-199.

World Bank, 2002. World Development Indicators. World Bank, Washington, DC. 